\title{
Chemical stability of Acid Rock Drainage (ARD) treatment sludge and implications for sludge management
}

\section{Environmental Science and Technology}

Prepared 13/10/2005

Danny M. McDonald*

Environmental Geoscience, La Trobe University, Victoria 3086 Australia

d.mcdonald@latrobe.edu.au

John A. Webb

Environmental Geoscience, La Trobe University, Victoria 3086 Australia

John.Webb@latrobe.edu.au

Jeff Taylor

Earth Systems, Suite 507, 1 Princess St. Kew, Victoria 3101 Australia

jeff.taylor@earthsystems.com.au

\section{Supporting information (7 tables and 3 figures over 11 pages)}


Table S1: Chemical analysis of treated water. See Table 1 for details on neutralisation process. $<$ dl $=$ less than detection limit, NA $=$ not analysed. Note K not analysed for all samples as initial results showed insignificant levels $(<0.4 \mathrm{mg} / \mathrm{L})$. A mid-range standard was analysed after every 5 samples; if it did not fall within $\pm 5 \%$ of the expected value, the instrument was recalibrated and samples reanalysed. A charge balance was calculated for each complete leachate analysis using the hydrochemical computer program Phreeqc; if the charge balance was outside $\pm 10 \%$, the sample was reanalysed for all elements.

\begin{tabular}{|c|c|c|c|c|c|c|c|c|c|c|c|c|}
\hline & $\begin{array}{c}\text { Treatmen } \\
\text { t pH }\end{array}$ & $\begin{array}{c}\mathrm{Fe} \\
(\mathrm{mg} / \mathrm{L})\end{array}$ & $\begin{array}{c}\mathrm{Al} \\
(\mathrm{mg} / \mathrm{L})\end{array}$ & $\begin{array}{c}\mathrm{Cu} \\
(\mathrm{mg} / \mathrm{L})\end{array}$ & $\begin{array}{c}\mathrm{Zn} \\
(\mathrm{mg} / \mathrm{L})\end{array}$ & $\begin{array}{c}\mathrm{Ca} \\
(\mathrm{mg} / \mathrm{L})\end{array}$ & $\begin{array}{c}\mathrm{Na} \\
(\mathrm{mg} / \mathrm{L})\end{array}$ & $\begin{array}{c}\mathrm{Mg} \\
(\mathrm{mg} / \mathrm{L})\end{array}$ & $\begin{array}{c}\mathrm{Si} \\
(\mathrm{mg} / \mathrm{L})\end{array}$ & $\begin{array}{c}\mathrm{Cl} \\
(\mathrm{mg} / \mathrm{L})\end{array}$ & $\begin{array}{c}\mathrm{SO}_{4} \\
(\mathrm{mg} / \mathrm{L})\end{array}$ & $\begin{array}{c}\text { CBE } \\
\%\end{array}$ \\
\hline Run 1 & 9.19 & $<\mathrm{dl}$ & 0.99 & $<\mathrm{dl}$ & $<\mathrm{dl}$ & 607 & 13.9 & 2.18 & NA & $<\mathrm{dl}$ & 1410 & 3.95 \\
\hline Run 2 & 8.85 & 1.14 & 0.45 & $<\mathrm{dl}$ & 0.11 & 657 & 4.70 & 3.32 & NA & $<\mathrm{dl}$ & 1540 & 2.48 \\
\hline Run 3 & 9.09 & 0.59 & $<\mathrm{dl}$ & $<\mathrm{dl}$ & $<\mathrm{dl}$ & 570 & NA & NA & NA & $<\mathrm{dl}$ & 1440 & -4.35 \\
\hline Run 4 & 9.24 & 0.95 & 0.67 & $<\mathrm{dl}$ & 0.07 & 581 & 6.74 & 4.26 & 0.38 & $<\mathrm{dl}$ & 1510 & -4.76 \\
\hline Run 5 & 9.18 & 0.66 & 0.31 & $<\mathrm{dl}$ & $<\mathrm{dl}$ & 642 & 6.32 & 3.28 & 0.15 & $<\mathrm{dl}$ & 1520 & 1.37 \\
\hline Run 6 & 8.33 & 0.22 & 0.16 & $<\mathrm{dl}$ & 0.14 & 328 & 363 & 567 & 1.41 & 45.0 & 3510 & 3.90 \\
\hline Run 7 & 8.37 & 0.52 & 0.85 & $<\mathrm{dl}$ & 0.15 & 630 & 10.3 & 4.88 & 1.41 & $<\mathrm{dl}$ & 1480 & 2.95 \\
\hline
\end{tabular}


Table S2: Chemical composition of neutralising reagents and ARD treatment sludges (wt\%) 1=XRF, 2=Acid Digest then AAS, 3=loss on drying $\left(1000^{\circ} \mathrm{C}\right.$ for 45 minutes). Units for neutralization potential (NP) are $\mathrm{kg} \mathrm{CaCO}_{3}$ equivalent/tonne dry sludge. See Table 1 for detail on run conditions. NA not analysed; $\mathrm{dl}<$ detection limit.

\begin{tabular}{|c|c|c|c|c|c|c|c|c|c|}
\hline & \multicolumn{2}{|c|}{ Neutralising reagent } & \multicolumn{7}{|c|}{ ARD treatment sludges } \\
\hline & KB-1 & Bauxsol & Run 1 & Run 2 & Run 3 & Run 4 & Run 5 & Run 6 & Run 7 \\
\hline Reagent & & & $\begin{array}{l}\text { Hydrated } \\
\text { Lime }\end{array}$ & $\begin{array}{l}\text { Hydrated } \\
\text { Lime }\end{array}$ & $\begin{array}{l}\text { limestone/ } \\
\text { Hydrated } \\
\text { Lime }\end{array}$ & $\begin{array}{c}\text { Limestone/ } \\
\text { Hydrated } \\
\text { Lime }\end{array}$ & KB-1 & Bauxsol & $\begin{array}{c}\text { Hydrated } \\
\text { Lime } \\
\text { (HDS) }\end{array}$ \\
\hline $\mathrm{SiO}_{2}{ }^{1}$ & 23.72 & 18.78 & 0.09 & 0.19 & 0.89 & 1.02 & 12.53 & 13.23 & 0.05 \\
\hline $\mathrm{TiO}_{2}{ }^{1}$ & 0.70 & 5.08 & $<\mathrm{dl}$ & $<\mathrm{dl}$ & 0.03 & 0.03 & 0.34 & 4.09 & 0.01 \\
\hline $\mathrm{Al}_{2} \mathrm{O}_{3}{ }^{1}$ & 11.50 & 15.33 & 2.62 & 2.64 & 1.96 & 2.65 & 8.16 & 14.41 & 2.47 \\
\hline $\mathrm{Fe}_{2} \mathrm{O}_{3}{ }^{1}$ & 1.70 & 23.70 & 21.77 & 21.65 & 13.31 & 18.33 & 18.4 & 33.07 & 20.09 \\
\hline $\mathrm{MnO}^{1}$ & 0.03 & 0.04 & 0.04 & 0.04 & 0.03 & 0.04 & 0.05 & 0.05 & 0.04 \\
\hline $\mathrm{MgO}^{1}$ & 1.17 & 9.44 & 0.16 & 0.12 & 0.41 & 0.41 & 0.68 & 1.32 & 0.29 \\
\hline $\mathrm{CaO}^{1}$ & 47.65 & 5.55 & 20.95 & 19.92 & 34.03 & 24.27 & 17.35 & 1.38 & 21.72 \\
\hline $\mathrm{Na}_{2} \mathrm{O}^{1}$ & 0.00 & 5.75 & 0.29 & 0.28 & 0.77 & 0.76 & 1.00 & 2.07 & 1.08 \\
\hline $\mathbf{K}_{2} \mathbf{O}^{1}$ & 0.39 & 0.37 & $<\mathrm{dl}$ & $<\mathrm{dl}$ & 0.16 & 0.17 & 0.27 & 0.29 & 0.06 \\
\hline $\mathbf{P}_{2} \mathbf{O}_{5}{ }^{1}$ & 0.06 & 0.13 & $<\mathrm{dl}$ & $<\mathrm{dl}$ & 0.02 & 0.02 & 0.04 & 0.11 & 0.01 \\
\hline $\mathrm{SO}_{3}{ }^{1}$ & 0.30 & 0.48 & 27.14 & 28.14 & 15.74 & 23.98 & 20.64 & 2.17 & 30.29 \\
\hline $\mathrm{CuO}^{2}$ & NA & $<0.4$ & 1.46 & 1.46 & 0.89 & 1.19 & 1.17 & 0.91 & 1.37 \\
\hline $\mathrm{ZnO}^{2}$ & NA & $<\mathrm{dl}$ & 1.44 & 1.43 & 0.87 & 1.15 & 1.15 & 0.89 & 1.25 \\
\hline $\mathbf{L O D}^{3}$ & 13.41 & 13.28 & 24.62 & 23.95 & 31.39 & 26.16 & 19.02 & 25.00 & 22.53 \\
\hline Total & 100.46 & 97.96 & 100.53 & 99.59 & 100.50 & 100.18 & 100.81 & 98.97 & 101.25 \\
\hline
\end{tabular}


Table S3: Mineralogy of neutralising reagents and ARD treatment sludges as determined by XRD. See Table 1 for more detail on run conditions.

\begin{tabular}{|c|c|c|c|c|c|c|c|c|c|c|c|}
\hline & \multicolumn{4}{|c|}{ Neutralising reagent } & \multicolumn{7}{|c|}{ ARD treatment sludge } \\
\hline & $\begin{array}{c}\text { Hydrated } \\
\text { Lime }\end{array}$ & Limestone & KB-1 & Bauxsol & Run 1 & Run 2 & Run 3 & Run 4 & Run 5 & Run 6 & Run 7 \\
\hline Amorphous & NA & NA & 59.0 & 31.2 & 40.3 & 40.6 & 30.1 & 39.3 & 55.9 & 56.7 & 16.1 \\
\hline Gypsum & & & & & 55.7 & 56.9 & 34.1 & 47.3 & 41.9 & & 82.2 \\
\hline Calcite & 5.5 & 98.2 & & 4.0 & 4.0 & 2.5 & 35.8 & 13.4 & & 0.8 & 1.7 \\
\hline Quartz & & & 2.3 & 4.0 & & & & & 2.2 & 2.3 & \\
\hline Hematite & & & & 38.2 & & & & & & 34.5 & \\
\hline Boehmite & & & & 1.6 & & & & & & 1.2 & \\
\hline Gibbsite & & & & 4.5 & & & & & & 2.8 & \\
\hline Anatase & & & & 2.2 & & & & & & 1.7 & \\
\hline $\begin{array}{l}\text { Lime } \\
\left(\mathrm{Ca}(\mathrm{OH})_{2}\right)\end{array}$ & 94.5 & & & 4.8 & & & & & & & \\
\hline Dolomite & & 1.7 & & & & & & & & & \\
\hline Illite & & 0.1 & & & & & & & & & \\
\hline $\begin{array}{l}\text { Quicklime } \\
\text { (CaO) }\end{array}$ & & & 38.7 & & & & & & & & \\
\hline Periclase & & & & 9.6 & & & & & & & \\
\hline
\end{tabular}


Table S4: Leachate analysis from deionised water extraction of ARD treatment sludges. Note K not analysed for all samples as initial results showed insignificant levels $(<0.4 \mathrm{mg} / \mathrm{L})$. A mid-range standard was analysed after every 5 samples; if it did not fall within $\pm 5 \%$ of the expected value, the instrument was recalibrated and samples reanalysed. A charge balance was calculated for each complete leachate analysis using the hydrochemical computer program Phreeqc; if the charge balance was outside $\pm 10 \%$, the sample was reanalysed for all elements.

\begin{tabular}{|c|c|c|c|c|c|c|c|c|c|c|c|c|}
\hline & \multicolumn{2}{|c|}{ Run 2} & \multicolumn{2}{|c|}{ Run 3} & \multicolumn{2}{|c|}{ Run 4} & \multicolumn{2}{|c|}{ Run 5} & \multicolumn{2}{|c|}{ Run 6} & \multicolumn{2}{|c|}{ Run 7} \\
\hline $\mathbf{p H}$ & 7.24 & 7.25 & 7.85 & 7.92 & 6.5 & 6.56 & 8.76 & 9.09 & 6.92 & 7.03 & 6.09 & 6.16 \\
\hline Fe (mg/L) & 0.22 & 0.33 & 0.54 & $<0.2$ & $<0.2$ & $<0.2$ & $<0.2$ & 0.2 & $<0.2$ & $<0.2$ & $<0.2$ & $<0.2$ \\
\hline Al (mg/L) & $<1.0$ & $<1.0$ & $<1.0$ & $<1.0$ & $<1.0$ & $<1.0$ & $<1.0$ & $<1.0$ & $<1.0$ & $<1.0$ & $<1.0$ & $<1.0$ \\
\hline Cu (mg/L) & $<0.4$ & $<0.4$ & $<0.4$ & $<0.4$ & $<0.4$ & $<0.4$ & $<0.4$ & $<0.4$ & $<0.4$ & $<0.4$ & $<0.4$ & $<0.4$ \\
\hline Zn (mg/L) & $<0.05$ & 0.07 & 0.82 & $<0.05$ & $<0.05$ & $<0.05$ & $<0.05$ & $<0.05$ & $<0.05$ & $<0.05$ & 0.06 & 0.11 \\
\hline Ca (mg/L) & 630 & 633 & 600 & 589 & 552 & 541 & 541 & 597 & 58.1 & 56.3 & 550 & 566 \\
\hline $\mathrm{Na}(\mathrm{mg} / \mathrm{L})$ & 2.28 & 16.6 & 4.56 & 3.92 & 1.16 & 1.09 & 2.18 & 2.00 & 44.4 & 45.2 & 0.93 & 0.89 \\
\hline Mg (mg/L) & 3.10 & 2.86 & 4.18 & 0.54 & 7.82 & 7.58 & 1.68 & 1.66 & 53.6 & 51.4 & 3.60 & 3.60 \\
\hline Si (mg/L) & $<1.0$ & $<1.0$ & $<1.0$ & $<1.0$ & $<1.0$ & $<1.0$ & $<1.0$ & $<1.0$ & $<1.0$ & $<1.0$ & 1.19 & 1.06 \\
\hline $\mathrm{SO}_{4}(\mathrm{mg} / \mathrm{L})$ & 1465 & 1466 & 1320 & 1437 & 1504 & 1358 & 1386 & 1403 & 465.5 & 448.5 & 1307 & 1323 \\
\hline CBE \% & 2.62 & 4.26 & 7.51 & -1.03 & -7.97 & -1.99 & -5.11 & 1.14 & -5.98 & -5.03 & 0.87 & 2.12 \\
\hline
\end{tabular}


Table S5: Leachate analysis from SPLP extraction of ARD treatment sludges. Note $\mathrm{K}$ not analysed for all samples as initial results showed insignificant levels $(<0.4 \mathrm{mg} / \mathrm{L})$. A mid-range standard was analysed after every 5 samples; if it did not fall within $\pm 5 \%$ of the expected value, the instrument was recalibrated and samples reanalysed. A charge balance was calculated for each complete leachate analysis using the hydrochemical computer program Phreeqc; if the charge balance was outside $\pm 10 \%$, the sample was reanalysed for all elements.

\begin{tabular}{|l|c|c|c|c|c|c|c|c|c|c|c|c|}
\hline & \multicolumn{2}{|c|}{ Run 2 } & \multicolumn{2}{c|}{ Run 3 } & \multicolumn{2}{c|}{ Run 4 } & \multicolumn{2}{c|}{ Run 5 } & \multicolumn{2}{c|}{ Run 6 } & \multicolumn{2}{c|}{ Run 7 } \\
\hline pH & 7.76 & 7.81 & 7.61 & 7.69 & 6.60 & 6.68 & 9.29 & 9.19 & 6.73 & 6.60 & 6.25 & 6.16 \\
\hline Fe (mg/L) & $<0.2$ & $<0.2$ & $<0.2$ & $<0.2$ & 0.29 & 0.41 & 1.89 & $<0.2$ & 1.16 & 1.71 & 0.67 & 0.67 \\
\hline Al (mg/L) & $<1.0$ & $<1.0$ & $<1.0$ & $<1.0$ & $<1.0$ & $<1.0$ & $<1.0$ & $<1.0$ & $<1.0$ & $<1.0$ & $<1.0$ & $<1.0$ \\
\hline Cu (mg/L) & $<0.4$ & $<0.4$ & 0.53 & 0.44 & $<0.4$ & $<0.4$ & $<0.4$ & $<0.4$ & $<0.4$ & $<0.4$ & $<0.4$ & $<0.4$ \\
\hline Zn (mg/L) & 0.34 & $<0.05$ & $<0.05$ & $<0.05$ & $<0.05$ & $<0.05$ & 0.15 & $<0.05$ & 0.25 & $<0.05$ & 0.28 & 0.22 \\
\hline Ca (mg/L) & 656 & 608 & 586 & 542 & 555 & 550 & 606 & 604 & 61.1 & 48.2 & 551 & 561 \\
\hline Na (mg/L) & 1.80 & 2.26 & 4.46 & 3.90 & 0.89 & 0.95 & 2.12 & 2.32 & 43.2 & 42.8 & 0.96 & 1.34 \\
\hline Mg (mg/L) & 2.90 & 2.96 & 4.34 & 4.26 & 7.72 & 7.88 & 1.62 & 1.66 & 51.8 & 57.0 & 3.52 & 3.62 \\
\hline Si (mg/L) & $<1.0$ & $<1.0$ & $<1.0$ & $<1.0$ & $<1.0$ & $<1.0$ & $<1.0$ & $<1.0$ & 1.33 & $<1.0$ & $<1.0$ & 1.06 \\
\hline SO4 (mg/L) & 1441 & 1427 & 1361 & 1356 & 1410 & 1497 & 1400 & 1402 & 466.4 & 454.6 & 1319 & 1326 \\
\hline CBE \% & 6.65 & 1.70 & 3.31 & -2.08 & -2.97 & -7.90 & 2.59 & 2.11 & -5.94 & -5.84 & 0.48 & 1.43 \\
\hline
\end{tabular}


Table S6: Leachate analysis from TCLP extraction of ARD treatment sludges. Note K not analysed for all samples as initial results showed insignificant levels $(<0.4 \mathrm{mg} / \mathrm{L})$. A mid-range standard was analysed after every 5 samples; if it did not fall within $\pm 5 \%$ of the expected value, the instrument was recalibrated and samples reanalysed.

\begin{tabular}{|c|c|c|c|c|c|c|c|c|c|c|c|c|c|c|}
\hline & \multicolumn{2}{|c|}{ Run 2} & \multicolumn{2}{|c|}{ Run 3} & \multicolumn{2}{|c|}{ Run 4} & \multicolumn{4}{|c|}{ Run 5} & \multicolumn{2}{|c|}{ Run 6} & \multicolumn{2}{|c|}{ Run 7} \\
\hline pH & 5.05 & 5.05 & 6.01 & 5.89 & 6.27 & 6.30 & 4.73 & 4.55 & 4.34 & 4.71 & 4.69 & 4.98 & 4.87 & 4.82 \\
\hline $\mathrm{Fe}(\mathrm{mg} / \mathrm{L})$ & 4.04 & 4.26 & 2.20 & 2.11 & $<0.2$ & $<0.2$ & 0.25 & 1.24 & 0.97 & 0.38 & 7.30 & 8.03 & 1.10 & 0.60 \\
\hline $\mathrm{Al}(\mathrm{mg} / \mathrm{L})$ & $<1.0$ & $<1.0$ & $<1.0$ & $<1.0$ & $<1.0$ & $<1.0$ & $<1.0$ & 2.01 & 12.54 & 6.79 & 256.2 & 250.0 & 3.82 & 5.01 \\
\hline Cu (mg/L) & 50.0 & 47.6 & 0.42 & 0.46 & 4.80 & 4.87 & 82.8 & 82.8 & 84.3 & 81.7 & 57.7 & 58.1 & 116 & 127 \\
\hline Zn (mg/L) & 146.4 & 138.8 & 18.46 & 17.72 & 75.00 & 75.80 & 155.0 & 116.8 & 121.4 & 143.2 & 186.8 & 190.8 & 235.0 & 219.4 \\
\hline Ca (mg/L) & 1226 & 1162 & 1897 & 1932 & 2106 & 2139 & 831 & 743 & 460 & 495 & 146 & 158 & 1271 & 1190 \\
\hline Na (mg/L) & 1.84 & 1.3 .0 & 4.20 & 5.34 & 1.07 & 0.78 & 1.86 & 1.60 & 2.88 & 3.34 & 358 & 360 & 1.06 & 1.23 \\
\hline Mg (mg/L) & 15.6 & 14.6 & 22.8 & 22.7 & 48.2 & 48.6 & 31.8 & 25.4 & 25.9 & 31.3 & 223 & 237 & 16.6 & 15.6 \\
\hline Si (mg/L) & $<1.0$ & $<1.0$ & $<1.0$ & $<1.0$ & 2.76 & 2.87 & 46.3 & 49.6 & $<1.0$ & $<1.0$ & 175 & 179 & 9.71 & 9.79 \\
\hline $\mathrm{SO}_{4}(\mathrm{mg} / \mathrm{L})$ & 1211 & 1188 & 894.8 & 902.2 & 974.1 & 927.3 & 1144 & 1248 & 1248 & 1202 & 234.4 & 246.7 & 1197 & 1188 \\
\hline
\end{tabular}


Table S7: Leachate analysis from SALT extraction of ARD treatment sludges. Note K not analysed for all samples as initial results showed insignificant levels $(<0.4 \mathrm{mg} / \mathrm{L})$. A mid-range standard was analysed after every 5 samples; if it did not fall within $\pm 5 \%$ of the expected value, the instrument was recalibrated and samples reanalysed. A charge balance was calculated for each complete leachate analysis using the hydrochemical computer program Phreeqc; if the charge balance was outside $\pm 10 \%$, the sample was reanalysed for all elements.

\begin{tabular}{|c|c|c|c|c|c|c|c|c|c|c|c|c|}
\hline & pH & $\begin{array}{c}\mathrm{Fe} \\
(\mathrm{mg} / \mathrm{L})\end{array}$ & $\begin{array}{c}\mathrm{Al} \\
(\mathrm{mg} / \mathrm{L})\end{array}$ & $\begin{array}{c}\mathrm{Cu} \\
(\mathrm{mg} / \mathrm{L})\end{array}$ & $\begin{array}{c}\mathrm{Zn} \\
(\mathrm{mg} / \mathrm{L})\end{array}$ & $\begin{array}{c}\mathrm{Ca} \\
(\mathrm{mg} / \mathrm{L})\end{array}$ & $\begin{array}{c}\mathrm{Na} \\
(\mathrm{mg} / \mathrm{L})\end{array}$ & $\begin{array}{c}\mathrm{Mg} \\
(\mathrm{mg} / \mathrm{L})\end{array}$ & $\begin{array}{c}\mathbf{S i} \\
(\mathrm{mg} / \mathrm{L})\end{array}$ & $\begin{array}{c}\mathrm{Cl} \\
(\mathrm{mg} / \mathrm{L})\end{array}$ & $\begin{array}{c}\mathrm{SO}_{4} \\
(\mathrm{mg} / \mathrm{L})\end{array}$ & CBE \% \\
\hline \multirow{6}{*}{ Run 2} & 5.88 & $<0.2$ & $<1.0$ & 1.76 & 47.8 & 620 & 2.96 & 18.7 & NA & $<1$ & 1499 & 4.37 \\
\hline & 3.78 & 4.71 & 23.62 & 217 & 223 & 529 & 3.06 & 17.2 & NA & $<1$ & 2173 & -1.09 \\
\hline & 2.64 & 77.5 & 160.2 & 269 & 251 & 475 & 2.16 & 16.8 & NA & $<1$ & 3271 & -2.22 \\
\hline & 2.34 & 492 & 198.1 & 281 & 266 & 444 & 2.80 & 16.6 & NA & $<1$ & 4617 & -5.46 \\
\hline & 2.25 & 1050 & 243.6 & 297 & 271 & 427 & 2.90 & 19.6 & NA & $<1$ & 5937 & -3.57 \\
\hline & 2.18 & 1610 & 265.4 & 301 & 278 & 409 & 2.04 & 17.8 & NA & $<1$ & 7411 & -6.00 \\
\hline \multirow{6}{*}{ Run 3} & 6.46 & 0.62 & $<1.0$ & 0.51 & 8.96 & 719 & 5.76 & 27.2 & NA & $<1$ & 1189 & -1.67 \\
\hline & 6.10 & 1.63 & $<1.0$ & 0.44 & 32.2 & 689 & 4.90 & 35.1 & NA & $<1$ & 1244 & -4.27 \\
\hline & 5.97 & 2.66 & $<1.0$ & $<0.4$ & 65.4 & 709 & 4.90 & 39.0 & NA & $<1$ & 1317 & -1.24 \\
\hline & 5.40 & 15.7 & $<1.0$ & 5.62 & 170 & 528 & 5.28 & 43.8 & NA & $<1$ & 1635 & -4.25 \\
\hline & 2.58 & 276 & 189.5 & 233 & 226 & 489 & 5.56 & 35.6 & NA & $<1$ & 4030 & -5.88 \\
\hline & 2.32 & 1730 & 250.4 & 250 & 243 & 454 & 4.80 & 48.8 & NA & $<1$ & 7725 & -0.71 \\
\hline
\end{tabular}




\begin{tabular}{|c|c|c|c|c|c|c|c|c|c|c|c|c|}
\hline & pH & $\begin{array}{c}\mathrm{Fe} \\
(\mathrm{mg} / \mathrm{L})\end{array}$ & $\begin{array}{c}\mathrm{Al} \\
(\mathrm{mg} / \mathrm{L})\end{array}$ & $\begin{array}{c}\mathrm{Cu} \\
(\mathrm{mg} / \mathrm{L})\end{array}$ & $\begin{array}{c}\mathrm{Zn} \\
(\mathrm{mg} / \mathrm{L})\end{array}$ & $\begin{array}{c}\mathrm{Ca} \\
(\mathrm{mg} / \mathrm{L})\end{array}$ & $\begin{array}{c}\mathrm{Na} \\
(\mathrm{mg} / \mathrm{L})\end{array}$ & $\begin{array}{c}\mathrm{Mg} \\
(\mathrm{mg} / \mathrm{L})\end{array}$ & $\begin{array}{c}\mathrm{Si} \\
(\mathrm{mg} / \mathrm{L})\end{array}$ & $\begin{array}{c}\mathrm{Cl} \\
(\mathrm{mg} / \mathrm{L})\end{array}$ & $\begin{array}{c}\mathrm{SO4} \\
(\mathrm{mg} / \mathrm{L})\end{array}$ & CBE \% \\
\hline \multirow{6}{*}{ Run 4} & 6.04 & $<0.2$ & $<1.0$ & 1.30 & 93.8 & 556 & 1.90 & 52.0 & $<1.0$ & $<1$ & 1494 & -0.51 \\
\hline & 4.31 & 1.09 & 35.64 & 280 & 376 & 461 & 1.38 & 70.2 & 13.2 & $<1$ & 2706 & -4.78 \\
\hline & 2.44 & 917 & 320.0 & 438 & 436 & 413 & 1.35 & 73.6 & 18.8 & $<1$ & 6922 & -1.91 \\
\hline & 2.30 & 191 & 392.2 & 436 & 430 & 375 & 1.15 & 72.4 & 18.5 & $<1$ & 9405 & 0.56 \\
\hline & 2.16 & 5280 & 517.8 & 459 & 456 & 358 & 0.69 & 71.2 & 26.7 & $<1$ & 16910 & 5.56 \\
\hline & 1.89 & 6340 & 535.4 & 482 & 464 & 303 & 1.32 & 71.0 & 27.1 & $<1$ & 20180 & 3.50 \\
\hline \multirow{6}{*}{ Run 5} & 5.96 & $<0.2$ & $<1.0$ & 1.18 & 34.8 & 532 & 2.54 & 23.2 & 10.7 & $<1$ & 1504 & -4.50 \\
\hline & 3.95 & $<0.2$ & 26.92 & 139 & 156 & 517 & 2.34 & 29.3 & 86.3 & $<1$ & 2136 & -7.28 \\
\hline & 3.76 & 2.72 & 55.02 & 215 & 233 & 471 & 2.60 & 4.56 & 132 & $<1$ & 2048 & 3.23 \\
\hline & 3.21 & 7.22 & 151.1 & 260 & 247 & 397 & 2.52 & 5.98 & 202 & $<1$ & 2923 & -9.96 \\
\hline & 2.34 & 850 & 319.2 & 204 & 195 & 385 & 2.42 & 6.76 & 202 & $<1$ & 5315 & -1.19 \\
\hline & 2.34 & 895 & 444.6 & 273 & 260 & 324 & 3.02 & 6.74 & 255 & $<1$ & 5920 & 2.20 \\
\hline \multirow{6}{*}{ Run 6} & 5.12 & 2.02 & 1.62 & 22.6 & 187 & 253 & 278 & 203 & 82.4 & 7.3 & 2439 & -4.30 \\
\hline & 3.90 & 5.71 & 340.8 & 181 & 286 & 171 & 350 & 272 & 274 & 12.2 & 5141 & -7.34 \\
\hline & 3.69 & 11.2 & 877.0 & 229 & 293 & 53.9 & 350 & 284 & 387 & 9.0 & 8204 & -9.72 \\
\hline & 3.46 & 42.9 & 1380 & 257 & 298 & 30.1 & 350 & 285 & 676 & 11.6 & 10930 & -7.61 \\
\hline & 2.87 & 410 & 1800 & 290 & 304 & 17.3 & 345 & 293 & 1160 & 8.0 & 13450 & -1.57 \\
\hline & 2.43 & 126 & 1970 & 313 & 316 & 6.38 & 355 & 302 & 1360 & 38.5 & 16230 & -1.38 \\
\hline
\end{tabular}




\begin{tabular}{|c|c|c|c|c|c|c|c|c|c|c|c|c|}
\hline & pH & $\begin{array}{c}\text { Fe } \\
(\mathrm{mg} / \mathrm{L})\end{array}$ & $\begin{array}{c}\mathrm{Al} \\
(\mathrm{mg} / \mathrm{L})\end{array}$ & $\begin{array}{c}\mathrm{Cu} \\
(\mathrm{mg} / \mathrm{L})\end{array}$ & $\begin{array}{c}\mathrm{Zn} \\
(\mathrm{mg} / \mathrm{L})\end{array}$ & $\begin{array}{c}\mathrm{Ca} \\
(\mathrm{mg} / \mathrm{L})\end{array}$ & $\begin{array}{c}\mathrm{Na} \\
(\mathrm{mg} / \mathrm{L})\end{array}$ & $\underset{(\mathrm{mg} / \mathrm{L})}{\mathrm{Mg}}$ & $\begin{array}{c}\mathbf{S i} \\
(\mathrm{mg} / \mathrm{L})\end{array}$ & $\begin{array}{c}\mathrm{Cl} \\
(\mathrm{mg} / \mathrm{L})\end{array}$ & $\begin{array}{c}\mathrm{SO4} \\
(\mathrm{mg} / \mathrm{L})\end{array}$ & CBE \% \\
\hline \multirow{6}{*}{ Run 7} & 5.86 & 0.21 & $<1.0$ & 0.61 & 47.4 & 556 & 0.89 & 10.7 & 2.88 & $<1$ & 1419 & -0.04 \\
\hline & 4.72 & $<0.2$ & 4.61 & 103 & 285 & 546 & 1.37 & 16.4 & 12.9 & $<1$ & 2037 & -2.49 \\
\hline & 3.80 & 1.91 & 115.2 & 325 & 326 & 461 & 0.97 & 16.7 & 27.0 & $<1$ & 3028 & -3.56 \\
\hline & 2.83 & 185 & 208.6 & 443 & 385 & 396 & 0.99 & 19.3 & 39.7 & $<1$ & 5386 & -3.17 \\
\hline & 2.60 & 710 & 286.4 & 469 & 399 & 408 & 0.82 & 19.1 & 42.6 & $<1$ & 8375 & -4.70 \\
\hline & 2.46 & 1680 & 326.0 & 475 & 426 & 404 & 0.83 & 19.2 & 45.5 & $<1$ & 12280 & -6.53 \\
\hline
\end{tabular}




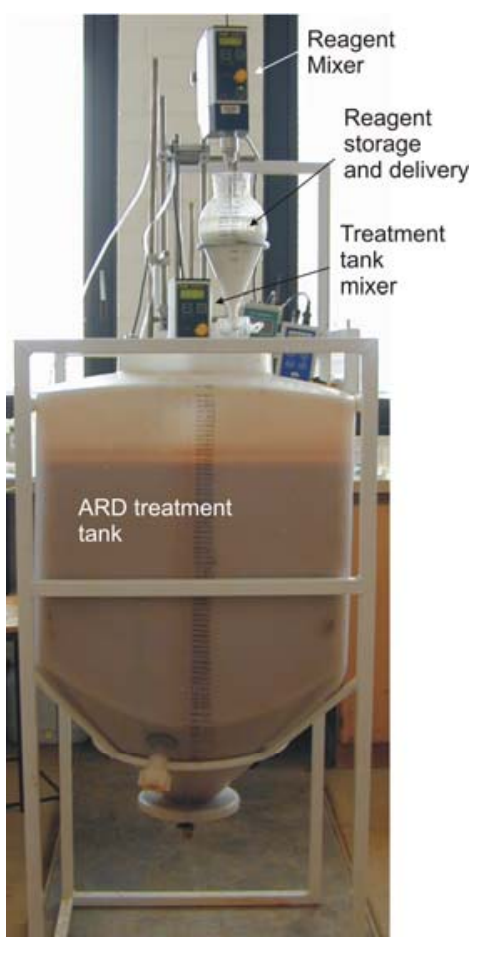

Figure S1: $170 \mathrm{~L}$ batch reactor with associated mixer and reagent delivery system used to produce sludge in Run 1-6

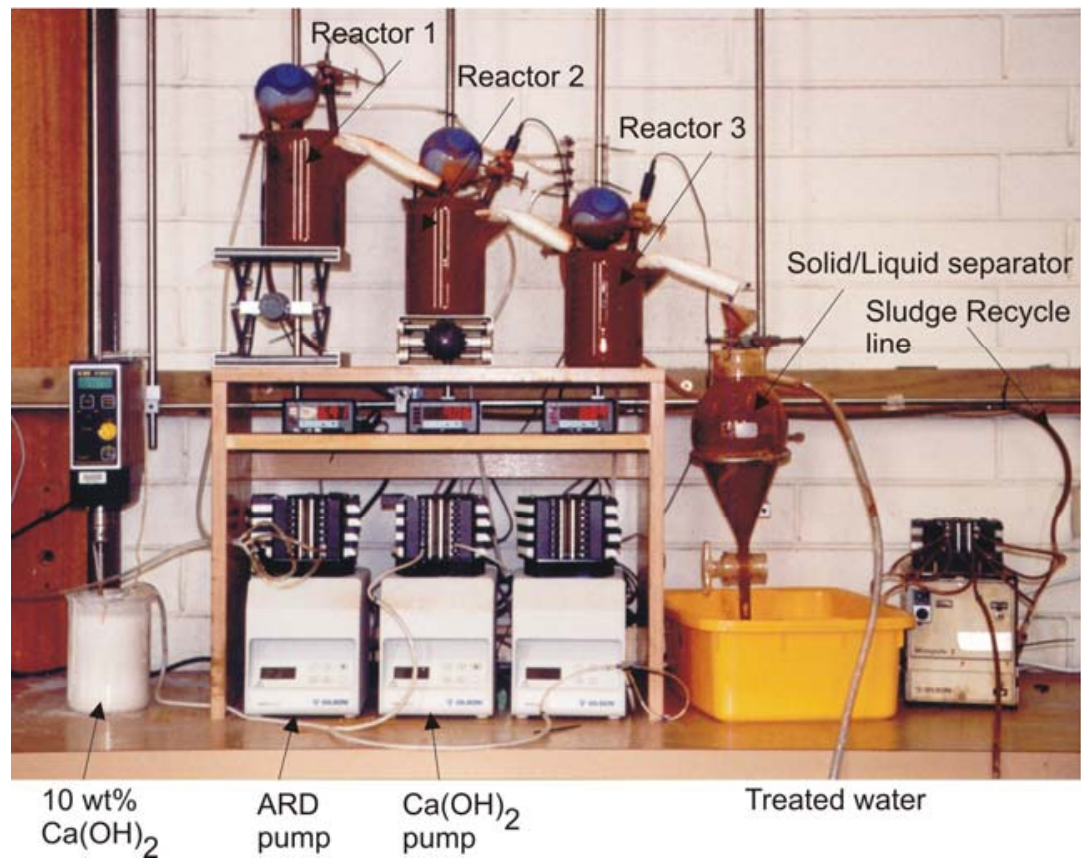

Figure S2: Laboratory scale HDS reactor used to produce sludge in Run 7 


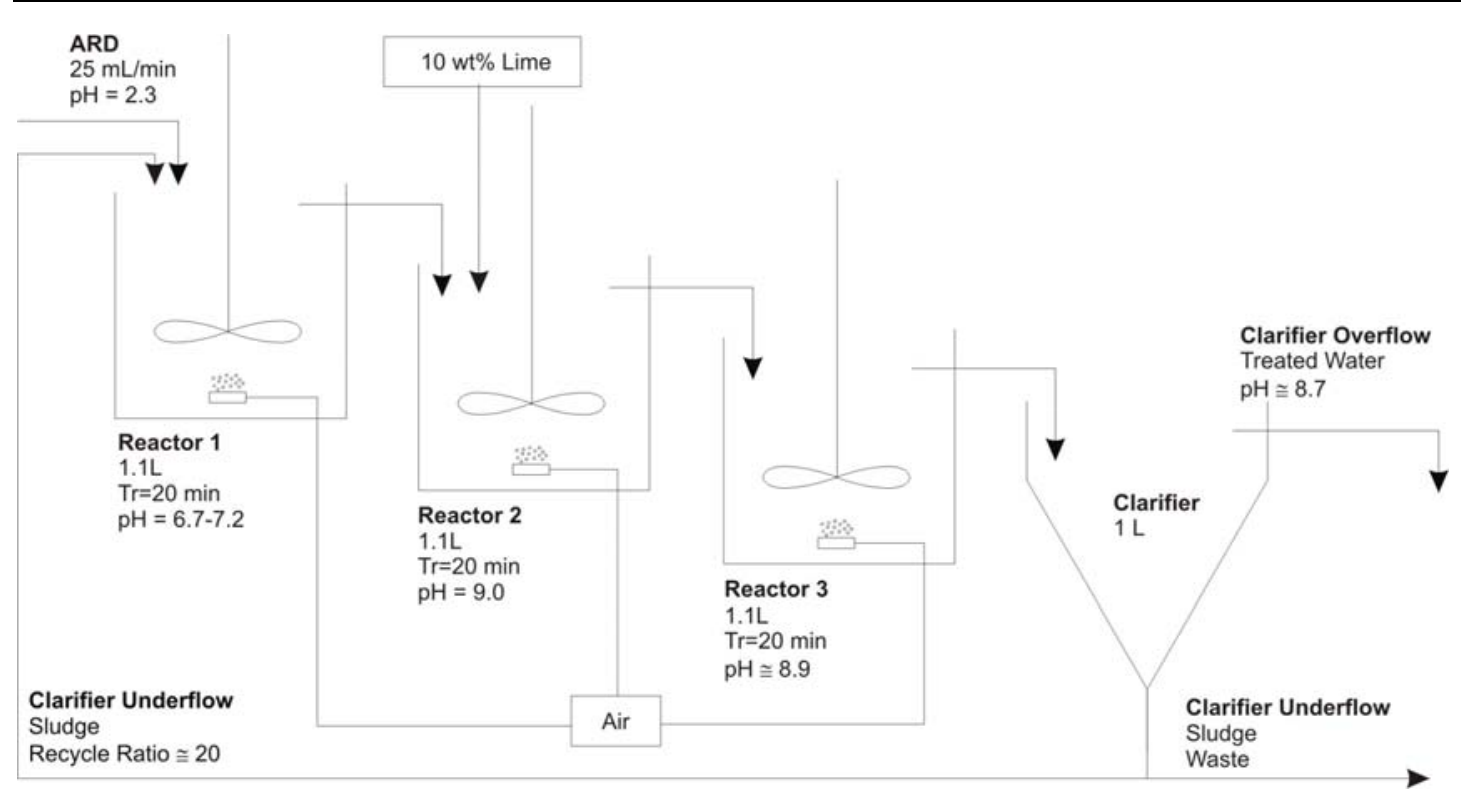

Figure S3: Schematic of laboratory scale HDS reactor. ( $\mathrm{Tr}=$ average retention time) 